\title{
Osteoarthritis prevalence and modifiable factors: a population study
}

Ronald Plotnikoff ${ }^{*}$, Nandini Karunamuni ${ }^{2}$, Ellina Lytvyak ${ }^{2}$, Christopher Penfold ${ }^{1}$, Donald Schopflocher ${ }^{2}$, Ikuyo Imayama ${ }^{3}$, Steven T. Johnson ${ }^{4}$ and Kim Raine ${ }^{2}$

\begin{abstract}
Background: This study's objectives were to investigate the prevalence of self-reported knee and hip osteoarthritis $(\mathrm{OA})$ stratified by age and sex and to examine the association of modifiable factors with knee and hip OA prevalence. The study was conducted using randomly sampled data gathered from four communities in the province of Alberta, Canada.

Methods: A large adult population sample $(N=4733)$ of individuals $\geq 18$ years were selected. Health-related information was collected through telephone interviews and community measurement clinics for which a sub-sample $(N=1808)$ attended. Participants self-reported OA during telephone interviews. Clinic interviews further assessed if the diagnosis was made by a health care professional. Statistical analyses compared prevalence of OA between sexes and across age categories. Associations between modifiable factors for OA and the prevalence of knee and hip OA were assessed using binary logistic regression modelling.

Results: Overall prevalence of self-reported OA in the total sample was $14.8 \%$, where $10.5 \%$ of individuals reported having knee OA and $8.5 \%$ reported having hip OA. Differences in prevalence were found for males and females across age categories for both knee and hip OA. In terms of modifiable factors, being obese (BMl $>30 \mathrm{~kg} / \mathrm{m} 2$ ) was significantly associated with the prevalence of knee (OR: 4.37; $95 \%$ Cl: 2.08,9.20) and hip (OR: 2.52; $95 \%$ Cl: 1.17,5.43) OA. Individuals who stand or walk a lot, but do not carry or lift things during their occupational activities were 2.0 times less likely to have hip OA (OR: 0.50; $95 \%$ Cl: 0.26,0.96). Individuals who usually lift or carry light loads or have to climb stairs or hills were 2.2 times less likely to have hip OA (OR: $0.45 ; 95 \% \mathrm{Cl}: 0.21,0.95)$. The odds of having hip OA were 1.9 times lower in individuals consuming recommended or higher vitamin C intake (OR: 0.52; $95 \% \mathrm{Cl}$ : 0.29,0.96). Significant differences in prevalence were found for both males and females across age categories.
\end{abstract}

Conclusion: The prevalence of knee and hip OA obtained in this study is comparable to other studies. Females have greater knee OA prevalence and a greater proportion of women have mobility limitations as well as hip and knee pain; it is important to target this sub-group.

Keywords: Knee osteoarthritis, Hip osteoarthritis, Prevalence, Risk-factors

\section{Background}

Osteoarthritis (OA) is the most common articular disease of the developed world and a leading cause of chronic disability, mostly as a consequence of knee OA and/or hip OA [1-3]. The economic costs of OA are high, including those related to treatment, for individuals and their families who must adapt their lives to the

\footnotetext{
*Correspondence: ronald.plotnikoff@newcastle.edu.au

'Priority Research Centre for Physical Activity and Nutrition, University of Newcastle, Callaghan, NSW 2308, Australia

Full list of author information is available at the end of the article
}

disease, and those due to lost work productivity [4, 5]. The prevalence of hand, knee, or hip joint OA has increased from 21 million in 1995 to an estimated 27 million among United States (US) adults [3]. Such increases are likely due to aging of the population and the rising prevalence of obesity [2].

OA has a multi-factorial etiology, with different sets of factors associated with its incidence $[1,5]$. Factors associated with OA have been broadly divided into personlevel factors and joint-level factors [2]. Person-level factors include age, sex, obesity, genetics, race/ethnicity 
and diet. Joint-level factors refer to factors that are unique to a particular joint such as, injury, activity, type of occupation, and muscle strength [2]. Factors associated with OA have also been classified as those that relate to $\mathrm{OA}$ development and those relating to disease progression. In terms of knee OA, Doherty [6], reports factors such as age, sex, occupation, weight status and recreational activity can play a role in the development of OA, and weight status and dietary factors may play a role in its progression.

When considering non-modifiable factors for OA, age and sex are the strongest predictors. For example, women are at greater risk for developing knee and hip OA compared to their male counterparts $[5,7,8]$. Hormonal factors, reduced volume of cartilage in the knee, and the fact that women are more likely to self-report have been considered as explanatory factors $[5,7,8]$.

Age is a significant contributor to the sex differences in prevalence of $\mathrm{OA}$, where females tend to have more severe knee and hand OA than men, particularly after menopausal age $[1,8]$. Age is one of the strongest non-modifiable factors for OA $[1,5]$, where this relationship is likely related to a combination of changes in the capacity for joint tissues to adapt to biomechanical stresses [2].

Obesity is a strong modifiable risk factor for the development of knee OA [1, 9], but less so for hip OA [2]. In a meta-analysis, those who were obese or overweight were nearly three times as likely to report knee OA [9]. The effects of obesity on OA are through both mechanical and systemic mechanisms. Obesity can exert an increased load as a consequence of increased body weight, however there may be differential systemic effects depending on the degree of fat versus lean mass [2, $10]$, involving the activity of adipocytokines [11].

Other modifiable factors of OA include occupation, dietary factors and physical activity [2, 5]. For example, repetitive joint loading through kneeling or squatting have been shown to be associated with an increased risk of knee OA [2, 12], and this risk is even greater for those who are overweight [2]. Furthermore, occupational lifting and prolonged standing have also been most strongly associated with hip OA $[2,12]$.

A number of studies have examined the role of vitamins (such as vitamins D and C) in OA [2, 13-16]. Mechanistically, it is thought that vitamin $C$ may serve to decrease cartilage loss in the joints while low vitamin $\mathrm{D}$ intake and reduced circulating serum vitamin $\mathrm{D}$ may confer an increased risk of knee OA [13].

The benefits of physical activity for OA are wellestablished [17], with national service organizations promoting active lifestyles, including walking for individuals with OA [18]. However, most individuals with knee OA do not meet recommended physical activity guidelines
[19]. Findings from a recent study has reported most people with knee OA are capable of walking at the recommended intensity needed to meet physical activity guidelines, and their knee pain has little impact on their level of physical activity [20].

Factors associated with OA could also interact in complex ways. For example, healthy lifestyle behaviours may reduce the age-related onset of $\mathrm{OA}$, and there can also be additional multifaceted associations between factors associated with OA. Considering the rising prevalence of $\mathrm{OA}$ in the population, identifying modifiable factors associated with $\mathrm{OA}$ is important to guide the development of effective interventions. Currently, there appears to be a paucity of data, particularly for Canada [21].

Using a large population sample consisting of random community samples, the objectives of this study were to: (i) investigate the prevalence of self-reported knee and hip OA stratified by age and sex; and, (ii) to examine the association of modifiable factors of body mass index (BMI), occupational factors, physical activity, vitamin $\mathrm{C}$ and D -intake, with self-reported knee OA and hip OA.

\section{Methods}

Data were gathered as part of the 2009-10 assessment period of the Healthy Alberta Communities (HAC) project, the details of this project and information on its sampling frame are reported elsewhere [22, 23]. Briefly, adults of four communities in the province of Alberta, Canada were randomly sampled (see Fig. 1). Data collection from the study participants took place in distinct phases. Phase 1 consisted of Computer Assisted Telephone Interviewing protocol, where survey data were gathered from 4733 individuals. From these individuals, a subsample of 1808 (38.2 \%) agreed to take part in community measurement clinics that collected clinical measures and additional self-reported health-related information (Phase 2). Inclusion criteria for the HAC project (Phase 1) were men and women of the intervention communities who were aged 18 years and older and not living in an institution. Out of these participants, pregnant women and persons in wheelchairs were excluded from Phase 2 of the study. Data from Phase 1 (telephone-based assessment) and Phase 2 (clinical-based assessments) are presented in the analysis of this paper.

\section{Ethics}

The study was approved by Research Ethics Boards at the University of Alberta. All data were collected directly from participants. Potential respondents to the telephone survey were informed of the purpose of the survey and asked to participate. Participation was completely voluntary. Agreement to participate served as verbal informed consent for Phase 1. For Phase 2 (i.e., subsample of those completing Phase 1), participants were asked to sign 


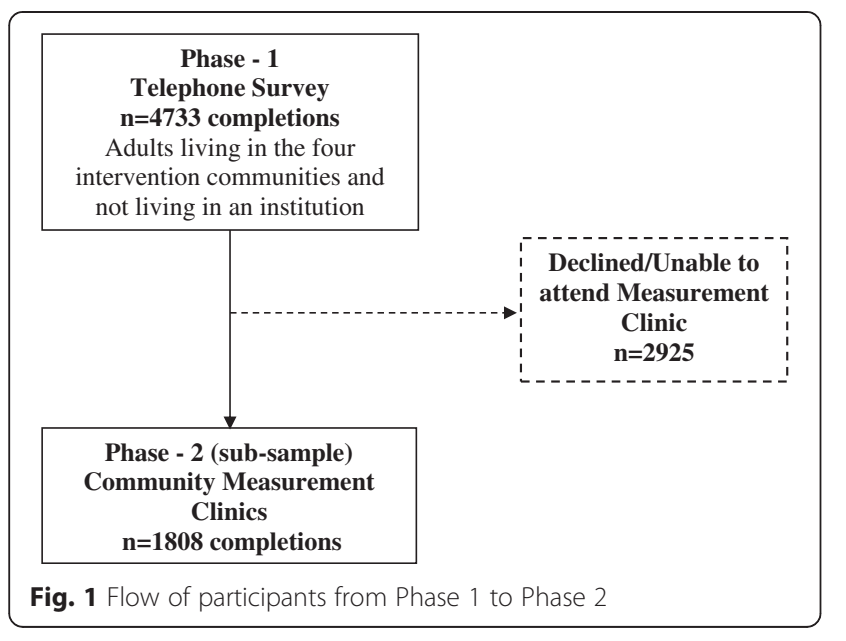

informed consent for the collection of physical measures and blood.

\section{Measures}

In Phase 1 of the study, during the phone interviews, participants were asked to report their age and sex, as well as to respond 'yes' or 'no' to the questions, "Do you have knee osteoarthritis?" and "Do you have hip osteoarthritis?" The options "Do not know" or "Refuse to answer" were also provided. This is a standard self-report measure employed in the Canadian Community Health Survey (CCHS) [24]. Categories of BMI were generated using participant self-reported height and weight estimates (objectively measured BMI was also obtained during the clinical assessments using a standardized protocol and equipment [23]). Physical activity level was assessed with a brief validated measure where participants were also asked to describe their main daily activities as: sit and don't walk; stand or walk quite a lot but do not carry or lift things; usually lift or carry light loads or have to climb stairs or hills often; do heavy work or carry very heavy loads [24]. The validated Godin-leisure time physical activity instrument, was employed to assess leisure-time physical activity [25]. Weekly frequencies of strenuous, moderate, and mild activities were multiplied by nine, five, and three, respectively, and then summed to obtain total weekly leisure activity scores [25]. Using validated cut-points, individuals having a weekly leisure activity score $\geq 24$ were classified as active for substantial health benefit and individuals with scores $\leq 23$ were classified as inactive [26].

In Phase 2 of the study (clinic interviews) participants ( $n=1808)$ answered 'yes' or 'no' to a five-item questionnaire relating to knee $\mathrm{OA}$ and mobility limitations, using a validated questionnaire [27]. Participants were asked: During the past 4 weeks, have you had knee pain on most days?; During the past 4 weeks, have you had knee pain while climbing down stairs or walking down slopes?; During the past 4 weeks, have you had swelling in one or both knees?; Do you have knee osteoarthritis?; If you do, was the diagnosis made by a rheumatologist or a general practitioner [27]? Further, participants responded 'yes' or 'no' to the following questions relating to hip OA and mobility limitations: During the past 4 weeks, have you had hip pain (groin or upper thigh) on most days?; During the past 4 weeks, have you had hip pain while climbing down stairs or walking down slopes?; During the past 4 weeks, have you noticed any limitation in the range of motion of one or both hips?; Do you have hip osteoarthritis? If you do, was the diagnosis made by a rheumatologist or a general practitioner [27]. Participants who responded 'yes' to (i) having OA through the phone survey, (ii) having OA through the clinic survey, and (iii) indicated their OA was diagnosed by a general practice physician or rheumatologist was coded as the study's robust indicator of OA.

During the Phase 2 assessments, Vitamin $C$ intake was estimated from the Block - Brief 2000 Food Frequency Questionnaire [28], and participants were categorised as meeting recommendations versus not meeting recommendations according to the daily intake of $<90 \mathrm{mg} /$ day for men; and $<75 \mathrm{mg} /$ day for women [29]. Vitamin D intake was also estimated from the Block - Brief 2000 Food Frequency Questionnaire and categorised as meeting versus not meeting the recommended daily intake of $600 \mathrm{IU} /$ day for 19-70 years, and <800 IU/day for 70+ years [30].

\section{Data analyses}

Descriptive statistics were calculated for all study variables. Age was stratified according to classification used in the National Health Interview Survey [31], using the categories: $18-44 ; 45-64$ and $\geq 65$ years. BMI was calculated as weight in kilograms divided by height in meters squared, and BMI categories were defined as: underweight/normal (BMI <25); overweight $(25 \leq \mathrm{BMI}<30)$; obese (30 $\leq$ BMI) [32].

Descriptive analysis was performed to identify the overall OA prevalence in the study sample, and the prevalence was stratified by age and sex for both knee and hip OA separately. Prevalence was also compared between sexes and across age categories.

Estimates of BMI from self-reported height and weight and the objectively measured height and weight were compared using Bland and Altman limits of agreement [33], as well as bivariate Pearson correlations. Among the sub-group of participants $(n=1808)$ who provided data from both phone interviews and community clinics, the reliability of self-reported OA through the singleitem phone interview was compared with the five-item clinic interview measure using Cohen's Kappa coefficient [34]. As suggested by Landis and Koch [35], a kappa of 0.40-0.75 was considered to represent intermediate to good agreement. 
Associations between modifiable factors associated with OA and the prevalence of knee and hip OA were assessed using binary logistic regression modelling [study objective (ii)]. The dependent outcome variable was prevalence of robust knee OA or hip OA constructed as a 'yes/no' dichotomous indicator. Based on existing literature identifying potential factors associated with $\mathrm{OA}$, independent explanatory variables for the multivariate models included: age, sex, weight status (BMI), occupational activity, leisure-time physical activity, vitamin C-intake and vitamin D-intake. Effects by age and sex were estimated using stratified logistic regression models. All the covariates included in our analyses had less than $5 \%$ missing data, with the exception of the leisure time physical activity measure which had $7.1 \%$ missing cases. Taking into account sufficiently large sample size, complete case analysis was applied [36-38].

Survey weights were calculated using year specific community age and sex counts supplied by the Alberta government for post-stratification. In addition, 300 bootstrap weights were generated to account for difference in sample characteristics (i.e., community of residence, age category, smoking, BMI, physical activity level, fruit and vegetable consumption, and self-reported health) between the Phase 1 and Phase 2 group. All analyses were performed using sample and bootstrap weights.

\section{Results}

Demographic characteristics of the sample are displayed in Table 1. The average age of the participants was 52.5 $( \pm 16.5)$ years and $55.2 \%$ of them had a university degree. Of the participants, $32.6 \%$ were male. The overall prevalence of self-reported OA (knee or hip) in the total sample $(N=4733)$ was $14.8 \%$. Out of these individuals, $10.5 \%$ self-reported having knee OA and $8.5 \%$ selfreported having hip OA with approximately $3 \%$ selfreported having both knee and hip OA. For knee OA, $2.1 \%$ gave the answer "Do not know" and $0.03 \%$ refused to answer. For hip OA, these values were 1.7 and $0.03 \%$ respectively.

Prevalence (weighted estimates) of both knee and hip OA stratified by age and sex are displayed in Table 2 . For knee OA, the prevalence was $6.3 \%$ for males and $8.9 \%$ for females $(\mathrm{t}=3.38 ; p=0.001)$. For hip OA, the prevalence was $4.4 \%$ for males and $7.6 \%$ for females $(\mathrm{t}=$ $4.64 ; p=0.001)$. For robust measures of knee and hip OA, obtained through clinic surveys, the prevalence of knee OA was $4.4 \%$ in males and $6.7 \%$ in females $(t=1.6 ; p=$ 0.112 ). For hip OA, the rates were 2.9 for males, and 4.1 for females $(t=1.0 ; p=0.325)$.

The reliability (Cohen's Kappa coefficient) of selfreported OA through the phone interview compared with the five-item measure during the clinic interview
Table 1 Demographic characteristics of the overall study sample $(N=4733)$

\begin{tabular}{|c|c|}
\hline & $\mathrm{N}(\%)$ or mean $\pm \mathrm{SD}$ \\
\hline \multicolumn{2}{|l|}{ Sex } \\
\hline Male & $1542(32.6)$ \\
\hline Female & $3189(67.4)$ \\
\hline Age, years & $52.5 \pm 16.5$ \\
\hline \multicolumn{2}{|l|}{ Education } \\
\hline - No university & $2095(44.5)$ \\
\hline - University & $2611(55.2)$ \\
\hline \multicolumn{2}{|l|}{ Sports participation ${ }^{a}$} \\
\hline - Weekly leisure activity score $\geq 24$ & $651(38.3)$ \\
\hline - Weekly leisure activity score $\leq 23$ & $1049(61.7)$ \\
\hline \multicolumn{2}{|l|}{ Daily activity } \\
\hline - Sit/not walk & $1099(23.6)$ \\
\hline - Stand/walk & $1988(42.7)$ \\
\hline - Light work load & $1304(28.0)$ \\
\hline - Heavy work loads & $270(5.8)$ \\
\hline $\begin{array}{l}\text { Level of combined household income } \\
\text { (before tax), above cut-off point }{ }^{b}\end{array}$ & $3700(86.5)$ \\
\hline Knee osteoarthritis self-reported & $482(10.5)$ \\
\hline Hip osteoarthritis self-reported & $395(8.5)$ \\
\hline Either knee or hip osteoarthritis self-reported & $679(14.8)$ \\
\hline BMI self-reported, $\left(\mathrm{kg} / \mathrm{m}^{2}\right)$ & $26.81 \pm 5.51$ \\
\hline BMI measured, $\left(\mathrm{kg} / \mathrm{m}^{2}\right)^{\mathrm{a}}$ & $28.28 \pm 5.98$ \\
\hline Overweight measured ${ }^{a}$ & $630(34.8)$ \\
\hline Obese measured $^{a}$ & $591(32.7)$ \\
\hline
\end{tabular}

Data are based on non-weighted estimates

${ }^{a}$ These measured variables were obtained during clinic interviews that were attended by 1808 participants

${ }^{\text {b}}$ The cut-off point for low-income is Low Income Cut-Offs (LICO) were calculated for each participant based on family size and belonging to the particular community [53]

was $k=0.73$ for knee $O A$, and $k=0.68$ for hip OA. Based on Phase 2 data, the correlations between the prevalence of: knee osteoarthritis and 'knee pain on most days' was $0.43(p<.01)$; knee osteoarthritis and knee swelling was $0.38(p<.01)$; and hip osteoarthritis and 'hip pain on most days' was $0.45 ;(p<.01)$. The average BMI obtained from self-reported height and weight compared to measured BMI was $26.8 \pm 5.5 \mathrm{~kg} /$ $\mathrm{m}^{2}$ and $28.3 \pm 6.0 \mathrm{~kg} / \mathrm{m}^{2}$ respectively. The mean difference between self-reported and measured BMIs was $-1.25 \mathrm{~kg} / \mathrm{m}^{2}$ (limits of agreement: -5.28 and 2.78). The Pearson correlations between these two measures was $r=0.93$.

Significant differences in prevalence were found for both males and females across age categories (see Table 3). Prevalence was significantly related to older age for both males and females for knee OA (females: $\chi^{2}=188.9, p<0.001$; males: $\left.\chi^{2}(2)=136.5 ; p<0.001\right)$ 
and for hip OA (females $\chi^{2}=238.8, p<0.001$; males $\left.X^{2}=104.2, p<0.001\right)$.

The results of assessments relating to OA and mobility limitations conducted during clinic interviews are presented in Table 3. Among females, for the age categories 18-44 years, 45-64 years and $>65$ years, 5.4, 9.0 and $6.1 \%$ reported having knee pain. The corresponding values for the three age groups having hip pain were 4.1, 9.3 and $7.4 \%$. The values for males for the same age categories were 8.2, 7.7 and $3.1 \%$ for knee pain and 2.6, 5.9 and $4.0 \%$ for hip pain (Table 3).

Results of binary logistic regression modelling for both knee and hip OA are displayed in Table 4 [study objective (ii)]. Among the overall sample, being obese (BMI $>30 \mathrm{~kg} / \mathrm{m}^{2}$ ) was significantly associated with the prevalence of knee (OR: 4.37; $95 \%$ CI: 2.08, 9.20; $p<0.001$ ) and hip (OR: 2.52; $95 \%$ CI: 1.17 , 5.43; $p=0.018)$ OA.
Among the overall sample, individuals who stand or walk quite a lot, but do not carry or lift things during their occupational activities were 2.0 times less likely to have hip OA (OR: 0.50; 95 \% CI: 0.26, 0.96; $p=0.038$ ). Further, persons who usually lift or carry light loads or have to climb stairs or hills were 2.2 times less likely to have hip OA (OR: 0.45; 95 \% CI: 0.21, 0.95; $p=0.037$ ). Additionally, the odds of having hip OA were 1.9 times lower in individuals consuming recommended or higher vitamin C intake (OR: 0.52; 95 \% CI 0.29, $0.96 ; p=0.035$ ).

Stratified models indicated that among females, being obese was significantly related with knee OA prevalence (OR: 6.72; 95 \% CI: 2.45, 18.45; $p<0.001$ ) and hip OA prevalence (OR: 6.67; $95 \%$ CI: 1.84, 24.21; $p=0.004$ ). The odds of hip OA were 3.35 times higher among females with vitamin D intakes at recommended levels or higher (OR: 3.35; 95 \% CI: 1.14, 9.88; $p=0.028$ ). None of

Table 2 Prevalence of knee and hip osteoarthritis by age and sex

\begin{tabular}{|c|c|c|c|}
\hline & Males $49.9 \%(N=2364)$ & Females $50.0 \%(N=2367)$ & Differential significance between sexes, $p$ \\
\hline \multicolumn{4}{|c|}{ Knee osteoarthritis $^{\mathrm{a}}$} \\
\hline All ages & $6.3(146)$ & $8.9(206)$ & 0.001 \\
\hline $18-44$ & $1.5(17)$ & $1.7(19)$ & 0.651 \\
\hline $45-64$ & $8.1(62)$ & $10.9(78)$ & 0.063 \\
\hline$>65$ & $18.8(61)$ & $24.3(97)$ & 0.069 \\
\hline \multicolumn{4}{|c|}{ Hip osteoarthritis ${ }^{\mathrm{a}}$} \\
\hline All ages & $4.4(102)$ & $7.6(176)$ & $<0.001$ \\
\hline $18-44$ & $1.3(15)$ & $0.7(7)$ & 0.119 \\
\hline $45-64$ & $4.7(36)$ & $8.5(61)$ & 0.003 \\
\hline$>65$ & $14.2(47)$ & $24.6(99)$ & $<0.001$ \\
\hline \multicolumn{4}{|c|}{ Knee osteoarthritis } \\
\hline All ages & $4.4(23)$ & $6.7(33)$ & 0.112 \\
\hline $18-44$ & $3.2(8)$ & $1.1(3)$ & 0.121 \\
\hline $45-64$ & $3.9(7)$ & $9.5(15)$ & 0.042 \\
\hline$>65$ & $10.3(8)$ & $17.2(14)$ & 0.210 \\
\hline \multicolumn{4}{|c|}{ Hip osteoarthritis ${ }^{\mathrm{b}}$} \\
\hline All ages & $2.9(15)$ & $4.1(20)$ & 0.325 \\
\hline $18-44$ & $2.8(7)$ & $0.3(1)$ & 0.030 \\
\hline $45-64$ & $2.0(4)$ & $5.5(9)$ & 0.090 \\
\hline$>65$ & $6.1(5)$ & $13.0(10)$ & 0.145 \\
\hline \multicolumn{4}{|c|}{ Knee OR Hip osteoarthritis } \\
\hline All ages & $6.5(34)$ & $8.8(43)$ & 0.173 \\
\hline $18-44$ & $5.1(12)$ & $1.5(3)$ & 0.025 \\
\hline $45-64$ & $5.9(11)$ & $12.0(19)$ & 0.049 \\
\hline$>65$ & $13.4(10)$ & $24.4(19)$ & 0.085 \\
\hline
\end{tabular}

Data are based on weighted estimates

a Values reported are self-reported data based on phone interviews

'Values reported are based on robust values. Robust values represent participants responding 'yes' to having OA through both the phone survey and clinic survey, and indicating their OA was diagnosed by a general practice physician or rheumatologist 
the variables were significant for the males, except for age. Level of physical activity was not significantly associated with knee or hip OA prevalence for either sex.

\section{Discussion}

The objectives of the current study were to investigate the prevalence of knee and hip OA and to examine the association of modifiable factors with knee and hip OA prevalence among a large population-based sample. Here we report the $14.8 \%$ prevalence of knee or hip OA by self-report is comparable to other studies. In the US, the overall OA prevalence is $13.9 \%$ for adults aged 25 and older [3]. A review of several investigations in Canada has reported the overall prevalence ranges between 7.5 and $14.7 \%$ [21].

In agreement with the current literature $[1,2]$, our study found that age is associated with the prevalence of both knee and hip OA for both males and females. The prevalence of OA was also higher among females, which is comparable to findings from other studies $[1,5,7,8]$. Further, a greater proportion of women had higher percentages on all six measures relating to mobility limitations, hip and knee pain, as well as swelling [3].

In terms of knee OA, females had greater prevalence compared to males which is consistent with the current literature. For example, in a review by Lawrence, and colleagues [3], knee OA prevalence among US women ranged from 4.9 to $18.7 \%$. In this review, some of the studies included older age groups (such as $\geq 60$ years), whereas our sample consisted of individuals $18+$, which may account for the lower proportions (i.e., younger individuals are less likely to be affected by OA) [3]. Differences in survey methods used, ethnicity and other sampling issues may also affect prevalence values across various surveys $[1,39]$.

Considering hip OA, our reported hip OA prevalences of 4.4 and $7.6 \%$ for men and women respectively are also comparable to the current literature. For instance, the Johnston County OA Project [40], that surveyed individuals $\geq 45$ years of age reported the rates for hip OA among men and women to be 8.7 and $9.3 \%$ respectively. A more recent study of individuals $\geq 60$ years of age living in Spain [41], documented a prevalence of $6.7 \%$ in men and $8.0 \%$ in women.

In our study, the robust measure (i.e., percentage of participants who responded 'yes' to having OA through both the phone survey and clinic survey, and indicated their OA was diagnosed by a general practice physician or rheumatologist), yielded lower prevalence for both knee and hip OA (than the single-item, self-report measures). The diagnosis of symptomatic radiographic OA by a physician or rheumatologist takes into consideration both structural change and joint pain or discomfort [1], whereas self-report measures may only involve subjective assessments of joint pain. Therefore, a lower prevalence when using the robust measure is somewhat expected. Further, when considering the study's robust measure, sex differences in prevalence was not significant for either knee or hip OA prevalence. According to the literature, self-report methods for detecting OA are

Table 3 Prevalence ${ }^{a}$ of $\mathrm{OA}$ and mobility limitations stratified by age and sex

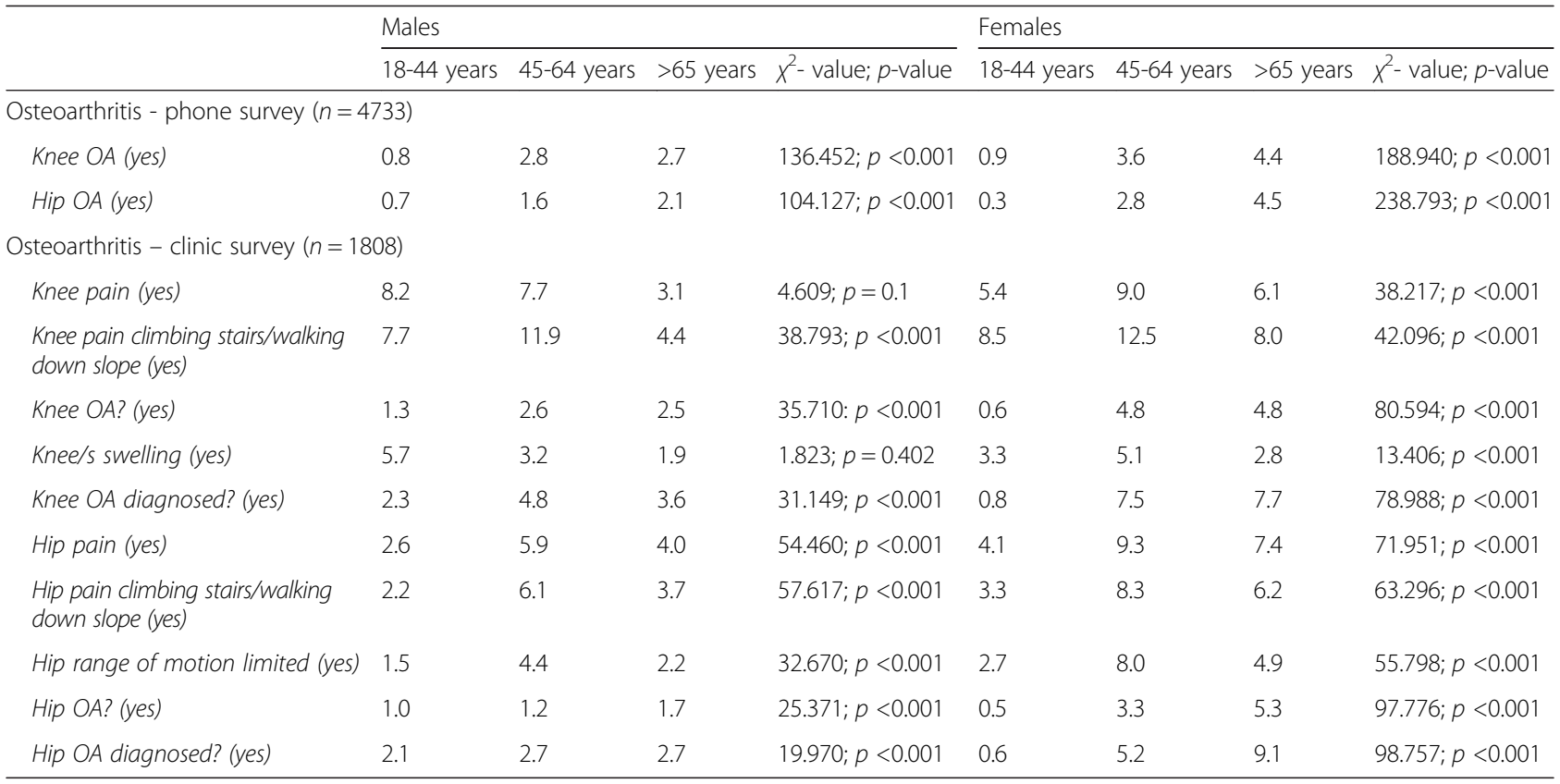

${ }^{\text {aPrevalence values are percentages }}$ 
Table 4 Binary logistic regression modelling of osteoarthritis

\begin{tabular}{|c|c|c|}
\hline \multirow[t]{2}{*}{ Total sample ${ }^{c}$} & \multicolumn{2}{|l|}{ Odds ratio $(95 \% \mathrm{Cl})$; p-value } \\
\hline & Knee osteoarthritis & Hip osteoarthritis \\
\hline \multicolumn{3}{|l|}{ BMI - clinic assessed } \\
\hline Under/ normal weight $(n=508)$ & - & - \\
\hline Overweight ( $n=547$ ) & $1.94(95 \%$ Cl: $0.87,4.32) ; p=0.105$ & 1.45 (95 \% Cl: $0.64,3.27) ; p=0.369$ \\
\hline Obese $(n=509)$ & 4.37 (95 \% Cl: $2.08,9.20) ; p<0.001$ & $2.52(95 \%$ Cl: $1.17,5.43) ; p=0.018$ \\
\hline \multicolumn{3}{|l|}{ Daily activity } \\
\hline Sit/not walk $(n=359)$ & - & - \\
\hline Stand/walk $(n=700)$ & 1.11 (95 \% Cl: 0.62, 2.00); $p=0.725$ & 0.50 (95\% Cl: $0.26,0.96) ; p=0.038$ \\
\hline Light work load $(n=433)$ & $0.72(95 \% \mathrm{Cl}: 0.36,1.44) ; p=0.354$ & 0.45 (95 \% Cl: $0.21,0.95) ; p=0.037$ \\
\hline Heavy workloads $(n=72)$ & 1.00 (95 \% Cl: 0.27, 3.69); $p=0.995$ & 1.51 (95 \% Cl: $0.47,4.79) ; p=0.488$ \\
\hline \multicolumn{3}{|l|}{ Sports } \\
\hline Weekly leisure activity (score $\leq 23, \%)(n=682)$ & - & - \\
\hline Weekly leisure activity (score $\geq 24, \%)(n=882)$ & 0.67 (95 \% Cl: $0.41,1.11) ; p=0.124$ & 1.21 (95\% Cl: $0.69,2.12) ; p=0.508$ \\
\hline \multicolumn{3}{|l|}{ Vitamin C - clinic interview ${ }^{a}$} \\
\hline Less $(n=928)$ & - & - \\
\hline Recommendation $(n=636)$ & 1.35 (95 \% Cl: $0.82,2.21) ; p=0.237$ & $0.52(95 \% \mathrm{Cl} 0.29,0.96) ; p=0.035$ \\
\hline \multicolumn{3}{|l|}{ Vitamin D - clinic interview } \\
\hline Less $(n=1401)$ & - & - \\
\hline Recommendation $(n=163)$ & 1.48 (95 \% Cl: $0.67,3.28) ; p=0.328$ & $1.73(95 \% \mathrm{Cl} 0.67,4.44) ; p=0.258$ \\
\hline \multicolumn{3}{|l|}{ Sex: Male ${ }^{d}$} \\
\hline \multicolumn{3}{|l|}{ BMI - clinic assessed } \\
\hline Under/ normal weight $(n=136)$ & - & - \\
\hline Overweight $(n=211)$ & 1.90 (95 \% Cl: 0.60, 6.08); $p=0.277$ & 0.81 (95\% Cl: $0.28,2.32) ; p=0.690$ \\
\hline Obese $(n=179)$ & $2.90(95 \% \mathrm{Cl}: 0.93,9.02) ; p=0.066$ & 0.99 (95 \% Cl: $0.35,2.82) ; p=0.992$ \\
\hline \multicolumn{3}{|l|}{ Daily activity } \\
\hline Sit/not walk $(n=142)$ & - & - \\
\hline Stand/walk $(n=214)$ & $1.42(95 \%$ Cl: $0.58,3.46) ; p=0.441$ & 0.59 (95 \% Cl: $0.22,1.65) ; p=0.317$ \\
\hline Light work load $(n=131)$ & $1.02(95 \% \mathrm{Cl}: 0.37,2.81) ; p=0.968$ & 0.45 (95\% Cl: $0.14,1.49) ; p=0.193$ \\
\hline Heavy workloads $(n=39)$ & $0.47(95 \% \mathrm{Cl}: 0.05,4.41) ; p=0.508$ & 1.32 (95 \% Cl: $0.34,5.17) ; p=0.691$ \\
\hline \multicolumn{3}{|l|}{ Sports } \\
\hline Weekly leisure activity (score $\leq 23, \%)(n=209)$ & - & - \\
\hline Weekly leisure activity (score $\geq 24, \%)(n=317)$ & 0.59 (95 \% Cl: $0.28,1.23) ; p=0.156$ & 1.43 (95\% Cl: $0.58,3.52) ; p=0.439$ \\
\hline \multicolumn{3}{|l|}{ Vitamin C - clinic interview ${ }^{a}$} \\
\hline Less $(n=337)$ & - & - \\
\hline Recommendation $(n=189)$ & 0.99 (95 \% Cl: 0.46, 2.15); $p=0.981$ & $0.48(95 \% \mathrm{Cl} 0.18,1.31) ; p=0.152$ \\
\hline \multicolumn{3}{|l|}{ Vitamin D - clinic interview ${ }^{b}$} \\
\hline Less $(n=489)$ & - & - \\
\hline Recommendation $(n=37)$ & 0.52 (95\% Cl: $0.07,3.73) ; p=0.512$ & $0.00 ; p=0.998$ \\
\hline \multicolumn{3}{|l|}{ Sex: Female ${ }^{d}$} \\
\hline \multicolumn{3}{|l|}{ BMI - clinic assessed } \\
\hline Under/ normal weight $(n=372)$ & - & - \\
\hline Overweight $(n=336)$ & $1.83(95 \%$ Cl: $0.59,5.71) ; p=0.295$ & 2.79 (95 \% Cl: $0.72,10.78) ; p=0.137$ \\
\hline Obese $(n=330)$ & $6.72(95 \% \mathrm{Cl}: 2.45,18.45) ; p<0.001$ & 6.67 (95\% Cl: 1.84, 24.21); $p=0.004$ \\
\hline
\end{tabular}


Table 4 Binary logistic regression modelling of osteoarthritis (Continued)

\begin{tabular}{|c|c|c|}
\hline \multicolumn{3}{|l|}{ Daily activity } \\
\hline Sit/not walk $(n=217)$ & - & - \\
\hline Stand/walk $(n=486)$ & 0.96 (95 \% Cl: 0.43, 2.15); $p=0.928$ & 0.53 (95 \% Cl: $0.21,1.30) ; p=0.165$ \\
\hline Light work load ( $n=302)$ & 0.55 (95 \% Cl: $0.21,1.43) ; p=0.219$ & 0.46 (95 \% Cl: $0.16,1.26) ; p=0.131$ \\
\hline Heavy workloads $(n=33)$ & 2.46 (95 \% Cl: $0.45,13.45) ; p=0.298$ & 1.74 (95 \% Cl: $0.18,17.29) ; p=0.635$ \\
\hline \multicolumn{3}{|l|}{ Sports } \\
\hline Weekly leisure activity (score $\leq 23, \%)(n=473)$ & - & - \\
\hline Weekly leisure activity (score $\geq 24, \%)(n=565)$ & 0.86 (95 \% Cl: $0.42,1.72) ; p=0.662$ & 1.08 (95 \% Cl: 0.50, 2.33); $p=0.848$ \\
\hline \multicolumn{3}{|l|}{ Vitamin C - clinic interview ${ }^{a}$} \\
\hline Less $(n=591)$ & - & - \\
\hline Recommendation $(n=447)$ & 1.73 (95 \% Cl: 0.88, 3.42); 0.113 & $0.61(95 \% \mathrm{Cl} 0.27,1.36) ; p=0.226$ \\
\hline \multicolumn{3}{|l|}{ Vitamin D - clinic interview ${ }^{\mathrm{b}}$} \\
\hline Less $(n=912)$ & - & - \\
\hline Recommendation $(n=126)$ & 2.07 (95 \% Cl: 0.82, 5.22); $p=0.121$ & 3.35 (95 \% Cl: 1.14, 9.88); $p=0.028$ \\
\hline \multicolumn{3}{|c|}{$\begin{array}{l}\text { The first-listed category for each variable is the reference category } \\
\text { Dependent variable was robust self-reported knee OA or hip OA. Robust values represent individual's self-reported OA (during phone interview and during clinic } \\
\text { interview), as well as being diagnosed as having OA during clinic interviews } \\
\text { a'Vitamin C recommendations were based on the Institute of Medicine (2006): adult males } 90 \mathrm{mg} / \text { day; adult females } 75 \mathrm{mg} / \mathrm{day} \\
\text { bVitamin D recommendations were based on the Institute of Medicine (2011): adults }<70 \text { years } 600 \mathrm{IU} / \text { day and adults } \geq 70 \text { years } 800 \mathrm{IU} / \text { day } \\
\text { Analyses adjusted for age and sex } \\
{ }^{c} \text { Analyses were controlled for age and sex } \\
\text { dAnalyses were controlled for age }\end{array}$} \\
\hline
\end{tabular}

known to exaggerate sex differences in prevalence due to reporting bias; women may be more likely to selfreport OA [8].

In our logistic regression analyses, for the overall sample and for females, being obese was strongly associated with knee and hip OA prevalence. Obesity is a wellestablished modifiable risk factor of knee OA [1, 9, 42]. The lifetime risk of developing symptomatic knee OA is approximately $40 \%$ in men and $47 \%$ in women, with higher risks among those who are obese [43], and studies have shown that decreasing BMI by two units or more over 10 years is associated with a $50 \%$ lower risk of developing symptomatic knee OA among women [44]. Further, duration of exposure to high BMI during adulthood confers risk of incident knee OA, suggesting the importance of weight control throughout life as a means of primary prevention of knee OA [45]. Although our study showed that obesity is also associated with hip $\mathrm{OA}$, according to the literature, the relationship between obesity and hip OA is weaker than with knee OA $[1,2]$.

Certain occupations have been shown to be associated with an increased risk of OA. Most studies report significantly greater risk of $\mathrm{OA}$ in individuals whose occupations involve activities with high physical demands [1, $2,12,46]$. Among occupational activity, our study found that individuals who spend time standing or walking, as well as lifting or carrying light loads and climb stairs or hills had a significantly lower risk of hip OA. While our study found no association for physically demanding work, the association of standing, walking and carrying light loads with a significantly lower risk of hip OA may indicate benefits of physical activity; considering that benefits of being active is well-established for OA [17]. However, we caution this interpretation considering our results cannot be used to infer cause and effect.

In our logistic regression model, physical activity was not associated with OA prevalence, which may be explained by the cross-sectional design. However, $61.7 \%$ of the participants reported being inactive (weekly leisure activity score $\leq 23$ ), whilst those with knee and hip OA were 79.0 and $75.7 \%$ inactive, respectively (compared to $58.5 \%$ of persons without OA being inactive). The percentage of inactive individuals we observed is higher than a US survey that found $44 \%$ of persons with arthritis were inactive (based on self-report measures) compared to $36 \%$ of adults without arthritis [47]. Our study's inactivity prevalence is concerning, considering physical activity can reduce pain, improve physical performance, as well as provide multiple other benefits of physical activity $[17,18]$.

In terms of dietary factors, the overall sample's odds of hip OA were 1.9 times lower in individuals consuming vitamin $\mathrm{C}$ intakes at or above recommended levels (<90 mg/day for men, $<75 \mathrm{mg} /$ day for women) [48]. While there have been conflicting results in terms of the effect of vitamin C on knee OA $[2,5,14,15]$, only a limited number of studies have specifically examined the relationship between hip $\mathrm{OA}$ and vitamin $\mathrm{C}$ intake. 
Engstrom, and colleagues [49], conducted a prospective population-based study that explored the relationship between intake of antioxidants and incidence of severe hip and/or knee OA, and reported that high dietary intake of vitamin $\mathrm{C}$ was significantly associated with the incidence of hip OA. However, in a 2-week crossover randomized trial, Jensen [50], found that pain was reduced during vitamin $\mathrm{C}$ treatment of individuals with radiographically verified $\mathrm{OA}$ of the knee and/or hip. Other studies have indicated no convincing evidence that vitamin $\mathrm{C}$ is effective in the treatment of any type of arthritis [51]. Further studies are needed to understand these conflicting findings.

In our study, the odds of hip OA were 3.4 times higher in females consuming recommended or higher vitamin D intakes (19-70 years <600 IU /day, 70+ years <800 IU/ day) [48]. Studies with specific focus on the association between vitamin D intake and hip OA are also very limited. One study that examined the relationship between serum vitamin D concentrations and incident radiographic hip OA among elderly women demonstrated that low serum levels of vitamin D may be associated with incident changes of radiographic hip OA characterized by joint space narrowing [52]. Our finding that only females are negatively affected by vitamin $\mathrm{D}$ is interesting and warrants further investigation.

\section{Strengths and limitations}

The strengths of this study include the use of a large random sample across four Canadian communities, and the use of validated measures. A further strength is the generalizability of the sample which was randomly selected in Phase 1. Further, population-based weights were included in the analyses and bootstrapping techniques were applied to enhance the population representativeness. Limitations of the study include its crosssectional design and the use of some self-report measures. However, Phase 2 five-item knee and hip OA measures had intermediate/good agreement with respective single-item measures from Phase 1 [35], and the selfreported and measured BMIs were highly correlated 0.93 . Another limitation of our study is that we were unable to collect information regarding the specific source (oral, written, $\mathrm{x}$-ray) of verification provided by their physician/rheumatologist. Additionally, assessing further factors associated with OA would have strengthened our study. For example, joint injury and resultant joint tissue destruction and loss could contribute to the development of OA [1]. Further, as vitamin D can also be obtained from sun exposure, it would have been beneficial to quantify the duration of participants' exposure to sunlight. Including these and other factors may have given more insight into the roles played by these modifiable factors. Despite the reported limitations, this study used a large representative population sample to estimate the prevalence of $\mathrm{OA}$ and examine in multivariate models a host of potential modifiable factors of OA. These results will help guide practice and future research.

\section{Conclusions}

The prevalence of knee and hip OA obtained in this study is comparable to other studies. Females have greater knee OA prevalence and a greater proportion of women have mobility limitations as well as hip and knee pain; it is important to target this sub-group.

\section{Competing interests}

The authors of this paper have no competing interests of any kind in regards to this manuscript.

\section{Authors' contributions}

RP conceived the study, participated in its design and coordination and helped to draft the manuscript. NK assisted in drafting the manuscript. EL and DS conducted the statistical analyses and assisted in drafting the manuscript; CP, II, and STJ assisted in drafting the manuscript. KR assisted with the co-ordination of the study and assisted in drafting the manuscript. All authors read and approved the final manuscript.

\section{Acknowledgements}

RP is supported by a Senior Research Fellowship Salary Award from the National Health and Medical Research Council (NHMRC), Australia. This study was funded by an Alberta Heritage Foundation for Medical Research (now called Alberta Innovates) Team Grant on Osteoarthritis (2008-2013).

\section{Author details}

${ }^{1}$ Priority Research Centre for Physical Activity and Nutrition, University of Newcastle, Callaghan, NSW 2308, Australia. ${ }^{2}$ School of Public Health, University of Alberta, Edmonton, AB, Canada. ${ }^{3}$ Fred Hutchinson Cancer Research Center Seattle, Washington, USA. ${ }^{4}$ Centre for Nursing and Health Studies, Athabasca University, Athabasca, AB, Canada.

Received: 21 August 2014 Accepted: 19 November 2015

Published online: 30 November 2015

\section{References}

1. Litwic A, Edwards MH, Dennison EM, Cooper C. Epidemiology and burden of osteoarthritis. Br Med Bull. 2013;105(1):185-99.

2. Neogi T, Zhang Y. Epidemiology of osteoarthritis. Rheum Dis Clin N Am. 2013;39(1):1-19.

3. Lawrence RC, Felson DT, Helmick CG, Arnold LM, Choi H, Deyo RA, et al. Estimates of the prevalence of arthritis and other rheumatic conditions in the United States: part II. Arthritis Rheum. 2008;58(1):26-35.

4. Altman RD. Early management of osteoarthritis. Am J Manag Care. 2010;16(2 Suppl):S41-7.

5. Chaganti RK, Lane N. Risk factors for incident osteoarthritis of the hip and knee. Curr Rev Musculoskelet Med. 2011;4(3):99-104.

6. Doherty M. Risk factors for progression of knee osteoarthritis. Lancet. 2001;358(9284):775-6.

7. Maleki-Fischbach $M$, Jordan JM. Sex differences in magnetic resonance imaging-based biomarkers and in those of joint metabolism. Arthritis Res Ther. 2010;12(4):1-8.

8. Srikanth VK, Fryer JL, Zhai G, Winzenberg TM, Hosmer D, Jones G. A meta-analysis of sex differences prevalence, incidence and severity of osteoarthritis. Osteoarthr Cartil. 2005;13(9):769-81.

9. Blagojevic M, Jinks C, Jeffery A, Jordan KP. Risk factors for onset of osteoarthritis of the knee in older adults: a systematic review and meta-analysis. Osteoarthr Cartil. 2010;18(1):24-33.

10. Koonce RC, Bravman JT. Obesity and osteoarthritis: more than just wear and tear. J Am Acad Orthop Surg. 2013;21(3):161-9.

11. Sowers MR, Karvonen-Gutierrez CA. The evolving role of obesity in knee osteoarthritis. Curr Opin Rheumatol. 2010;22(5):533. 
12. Yoshimura N, Sasaki S, Iwasaki K, Danjoh S, Kinoshita H, Yasuda T, et al. Occupational lifting is associated with hip osteoarthritis: a Japanese case-control study. J Rheumatol. 2000;27(2):434-40.

13. McAlindon TE, LaValley M, Schneider E, Nuite M, Lee J, Price L, et al. Effect of vitamin D supplementation on progression of knee pain and cartilage volume loss in patients with symptomatic osteoarthritis: a randomized controlled trial. JAMA. 2013;309(2):155-62.

14. Peregoy J, Wilder FV. The effects of vitamin C supplementation on incident and progressive knee osteoarthritis: a longitudinal study. Public Health Nutr. 2010;14(04):709.

15. McAlindon TE, Biggee BA. Nutritional factors and osteoarthritis: recent developments. Curr Opin Rheumatol. 2005;17(5):647-52.

16. Chaganti RK, Tolstykh I, Javaid M, Neogi T, Torner J, Curtis J, et al. Association of baseline vitamin C with incident and progressive radiographic knee OA. The MOST study. Arthritis Rheum. 2008;58(9):S897.

17. Conaghan PG, Dickson J, Grant RL. Guidelines: care and management of osteoarthritis in adults: summary of NICE guidance. BMJ: Br Med J. 2008; 336(7642):502

18. Arthritis: intervention programs. [http://www.cdc.gov/arthritis/interventions.htm]

19. Dunlop DD, Song J, Semanik PA, Chang RW, Sharma L, Bathon JM, et al. Objective physical activity measurement in the osteoarthritis initiative: are guidelines being met? Arthritis Rheum. 2011;63(11):3372-82

20. White DK, Tudor-Locke C, Felson DT, Gross KD, Niu J, Nevitt M, et al. Do radiographic disease and pain account for why people with or at high risk of knee osteoarthritis do not meet physical activity guidelines? Arthritis Rheum. 2013;65(1):139-47.

21. Prevalence of Arthritis and Rheumatic Diseases around the World. A Growing Burden and Implications for Health Care Needs. [http://www.modelsofcare.ca/ pdf/10-02.pdf].

22. Raine KD, Plotnikoff R, Schopflocher D, Lytvyak E, Nykiforuk Cl, Storey K, et al. Healthy Alberta Communities: impact of a three-year community-based obesity and chronic disease prevention intervention. Prev Med. 2013;57(6):955-62.

23. Raine KD, Plotnikoff R, Nykiforuk C, Deegan H, Hemphill E, Storey K, et al. Reflections on community-based population health intervention and evaluation for obesity and chronic disease prevention: the Healthy Alberta Communities project. Int J Public Health. 2010;55(6):679-86.

24. Statistics Canada. 2006 Canadian Community Health Survey (CCHS) Cycle 3. 1. Public Use Microdata File (PUMF) User Guide. In: Statistics Canada (Ed.). 2005.

25. Godin G, Shephard RJ. Gender differences in perceived physical self-efficacy among older individuals. Percept Mot Skills. 1985;60(2):599-602.

26. Godin G. The Godin-Shephard leisure-time physical activity questionnaire. Health Fitness J Canada. 2011:4(1):18-22.

27. Roux C, Saraux A, Mazieres B, Pouchot J, Morvan J, Fautrel B, et al. Screening for hip and knee osteoarthritis in the general population: predictive value of a questionnaire and prevalence estimates. Ann Rheum Dis. 2008;67(10):1406-11.

28. Block G, Gillespie C, Rosenbaum EH, Jenson C. A rapid food screener to assess fat and fruit and vegetable intake. Am J Prev Med. 2000;18(4):284-8.

29. Institute of Medicine. Dietary reference intakes: the essential guide to nutrient requirements. Washington: The National Academies Press; 2006.

30. Institute of Medicine. Dietary reference intakes for calcium and vitamin D. Washington: The National Academies Press; 2011.

31. Hootman J, Bolen J, Helmick C, Langmaid G. Prevalence of doctordiagnosed arthritis and arthritis-attributable activity limitationâ€"United States, 2003-2005. MMWR Morb Mortal Wkly Rep. 2006:55(40):1089-92.

32. World Health Organization. Global database on body mass index. 2006

33. Bland JM, Altman DG. Measuring agreement in method comparison studies. Stat Methods Med Res. 1999:8(2):135-60

34. Cohen J. A coefficient of agreement for nominal scales. Educ Psychol Meas. 1960:20(1):37-46.

35. Landis JR, Koch GG. The measurement of observer agreement for categorical data. Biometrics. 1977;33(1):159-74.

36. Acuria $E$, Rodriguez $C$, editors. The treatment of missing values and its effect on classifier accuracy. Berlin: Springer; 2004.

37. Dong Y, Peng CY. Principled missing data methods for researchers. SpringerPlus. 2013:2(1):222

38. Little R, Rubin DB. Statistical analysis with missing data. 2nd ed. John Wiley \& Sons, Inc: Hoboken, NJ: 2002.

39. Pereira D, Peleteiro B, Araujo J, Branco J, Santos R, Ramos E. The effect of osteoarthritis definition on prevalence and incidence estimates: a systematic review. Osteoarthr Cartil. 2011;19(11):1270-85.
40. Helmick C, Renner JB, Luta G, Dragomir A, Kalsbeek WD, Abbate L. Prevalence of hip pain, radiographic hip osteoarthritis $(O A)$, severe radiographic hip OA, and symptomatic hip OA: the Johnson County Osteoarthritis Project. Arthritis Rheum. 2003:48 Suppl 9:S212.

41. Quintana JM, Arostegui I, Escobar A, Azkarate J, Goenaga JI, Lafuente I. Prevalence of knee and hip osteoarthritis and the appropriateness of joint replacement in an older population. Arch Intern Med. 2008;168(14):1576.

42. Grotle M, Hagen KB, Natvig B, Dahl FA, Kvien TK. Obesity and osteoarthritis in knee, hip and/or hand: an epidemiological study in the general population with 10 years follow-up. BMC Musculoskelet Disord. 2008;9(1):132.

43. Murphy L, Schwartz TA, Helmick CG, Renner JB, Tudor G, Koch G, et al. Lifetime risk of symptomatic knee osteoarthritis. Arthritis Care Res. 2008;59(9):1207-13.

44. Felson DT, Zhang Y, Anthony JM, Naimark A, Anderson JJ. Weight loss reduces the risk for symptomatic knee osteoarthritis in womenThe Framingham Study. Ann Intern Med. 1992;116(7):535-9.

45. Wills AK, Black S, Cooper R, Coppack RJ, Hardy R, Martin KR, et al. Life course body mass index and risk of knee osteoarthritis at the age of 53 years: evidence from the 1946 British birth cohort study. Ann Rheum Dis 2012;71(5):655-60.

46. Richmond SA, Fukuchi RK, Ezzat A, Schneider K, Schneider G, Emery CA. Are joint injury, sport activity, physical activity, obesity, or occupational activities predictors for osteoarthritis? A systematic review. J Orthop Sports Phys Ther. Forthcoming 2013

47. Shih M, Hootman JM, Kruger J, Helmick CG. Physical activity in men and women with arthritis: National Health Interview Survey, 2002. Am J Prev Med. 2006;30(5):385-93.

48. Fischer DV, Bryant J, Fischer DV, Bryant J. Effect of certified personal trainer services on stage of exercise behavior and exercise mediators in female college students. J Am Coll Health. 2008;56(4):369-76.

49. Engstrom G, De Verdier MG, Nilsson P. Incidence of severe knee and hip osteoarthritis in relation to dietary intake of antioxidants beta-carotene, vitamin C, vitamin E and selenium: a population-based prospective cohort study. Arthritis Rheum. 2009;60:s235-6.

50. Jensen $\mathrm{NH}$. Reduced pain from osteoarthritis in hip joint or knee joint during treatment with calcium ascorbate. A randomized, placebo-controlled cross-over trial in general practice. Ugeskr Laeger. 2003;165(25):2563-6.

51. Canter P, Wider B, Ernst E. The antioxidant vitamins A, C, E and selenium in the treatment of arthritis: a systematic review of randomized clinical trials. Rheumatology. 2007;46(8):1223-33.

52. Lane NE, Gore R, Cummings SR, Hochberg MC, Scott JC, Williams EN, et al. Serum Vitamin D levels and incident changes of radiographic hip osteoarthritis. Arthritis Rheum. 1999;42(5):854-60.

53. Statistics Canada. Income Statistics Division: low income lines, 2009-2010 (Amended June 20, 2011). Ottawa: Statistics Canada; 2011.

\section{Submit your next manuscript to BioMed Central and we will help you at every step:}

- We accept pre-submission inquiries

- Our selector tool helps you to find the most relevant journal

- We provide round the clock customer support

- Convenient online submission

- Thorough peer review

- Inclusion in PubMed and all major indexing services

- Maximum visibility for your research

Submit your manuscript at www biomedcentral com/submit 